\title{
L'empêchement au travail : une source majeure de TMS ?
}

Impediment at work: a major source of musculoskeletal disorders

Laerte Idal Sznelwar, Fausto Leopoldo Mascia et Gilbert Bouyer

\section{(2) OpenEdition}

\section{Journals}

Édition électronique

URL : http://journals.openedition.org/activites/1339

DOI : 10.4000/activites.1339

ISSN : $1765-2723$

\section{Éditeur}

ARPACT - Association Recherches et Pratiques sur les ACTivités

\section{Référence électronique}

Laerte Idal Sznelwar, Fausto Leopoldo Mascia et Gilbert Bouyer, «L'empêchement au travail : une source majeure de TMS? », Activités [En ligne], 3-2 I octobre 2006, mis en ligne le 15 octobre 2006, consulté le 02 mai 2019. URL : http://journals.openedition.org/activites/1339; DOI : 10.4000/ activites.1339

\section{(c) $(i)(9)$}

Activités est mis à disposition selon les termes de la licence Creative Commons Attribution - Pas d'Utilisation Commerciale - Pas de Modification 4.0 International. 


\title{
L'empêchement au travail : une source majeure de TMS ?
}

\author{
Laerte Idal Sznelwar \\ Fausto Leopoldo Mascia \\ Gilbert Bouyer \\ Universidade de São Paulo, Escola Politécnica \\ Departamento de Engenharia de Produção \\ Av. Prof. Almeida Prado, 531 - Cidade Universitária \\ 05508-900, São Paulo - SP - Brasil. \\ laertesz@usp.br ; fmascia@usp.br ; learn10@uol.com.br
}

\begin{abstract}
Impediment at work: a major source of musculoskeletal disorders. The discussion about the genesis of musculoskeletal disorders is partly based on the question of movement, speed, force and repetitiveness. From various studies relating to the WRMD concerning work situations in the services sector, we propose a discussion based on the idea of impediment. The analysis is centered on the effort not to move, the effort not to think, the effort not to feel, taking into account the aspects of work organization and the contents of the task which are true impediments for the subject
\end{abstract}

\section{KEYWORDS}

Impediment, musculoskeletal disorders, ergonomic work analysis, call center.

\section{1.- Introduction}

Cet article propose de discuter des résultats issus d'études consécutives à l'apparition de troubles musculo-squelettiques dans diverses situations de travail à partir du concept d'empêchement. Dans les études les plus traditionnelles de l'ergonomie de l'activité, on a démontré que, en vue de la réalisation du travail, les sujets construisent un compromis avec les contraintes de la tâche, modifiant celle-ci dans certaines limites pour pouvoir atteindre les objectifs fixés par l'entreprise (Guérin, Laville, Daniellou, Duraffourg, \& Kerguelen, 2001). Ce compromis, qui est mobile à l'intérieur de certaines limites, peut être considéré comme la construction, par les sujets, d'une relation dialectique entre la recherche des résultats et leur santé. Il reviendrait à l'action ergonomique de proposer des solutions qui permettent la construction de ce compromis, favorisant ainsi non seulement l'atteinte des résultats attendus dans le cadre de la production mais aussi la protection de la santé des travailleurs.

Toutefois, il existe un grand nombre de situations de travail où ces compromis sont impossibles : l'atteinte du but s'effectue au détriment de la santé de l'opérateur. Dans ce cas, on se trouve face à des aspects de l'organisation du travail et du contenu de la tâche qui sont de véritables empêchements pour le sujet.

Par empêchement (action d'empêcher), on entend un processus qui rend l'action difficile, la paralyse, 
la bloque, qui obstrue, inhibe ou fait obstacle à l'exécution d'une action. Le concept d'empêchement s'applique quand le sujet n'a pas d'autre choix sinon celui de céder, de s'arrêter de faire, de s'arrêter de « fonctionner », de s'arrêter « d'être ».

Dans ce texte, on argumente que la genèse des TMS est liée aux empêchements rencontrés dans l'activité. Mais on voudrait franchir une étape supplémentaire. Les TMS traduisent les empêchements au plan du corps. Mais l'empêchement concerne également les sphères psychologiques et sociales (par exemple les processus d'identification et de reconnaissance du sujet). L'empêchement est donc, certes, une source de TMS, mais il est surtout à l'origine d'une dégradation de la santé au sens le plus large, dans ses dimensions physique, psychique et sociale.

La discussion a été inspirée par le fait que, lors de l'analyse de différentes situations de travail du secteur bancaire financier, nous avons rencontré une quantité significative de personnes qui exprimaient un type de souffrance liée au travail, à commencer par des douleurs passagères, incommodités, et inconforts, allant jusqu'à des formes graves de troubles musculo-squelettiques (TMS) et de troubles psychiques. D'une manière générale, cette souffrance est analysée comme étant le résultat d'activités répétitives effectuées sous forte contrainte de temps. Dans certaines situations, les troubles ostéomusculaires reçoivent la référence de maladies d'hyper sollicitation.

Pour mener cette discussion, on s'appuie sur des concepts développés dans des domaines disciplinaires distincts. Pour ce qui est du fonctionnement du corps, on s'appuie sur la physiologie traditionnelle. Pour ce qui est de la réalisation des actions et de la dimension psychique, on s'appuie sur la clinique du travail (psychodynamique du travail et clinique de l'activité). Les résultats qui seront présentés sont issus d'études réalisées auprès de travailleurs de centres d'appel (banques, administration de cartes de crédit, téléphonie). Les méthodes d'analyse sont l'Analyse Ergonomique de l'Activité et l'Action en Psychodynamique du Travail.

\section{2.- Questionnement initial}

D'une manière générale, on peut définir le travail comme une action, comme un « faire », comme un engagement du sujet dans la réalisation de quelque chose, qui peut être tangible ou pas. Mais on peut aussi centrer l'analyse du travail sur le « ne pas faire », sur tout ce qu'il ne faut pas faire pour pouvoir travailler et produire. Ce fait a déjà été souligné par Clot (1999).

Il faut néanmoins faire une distinction entre l'attention consacrée à une action, centrée sur quelque chose que nous sommes en train d'exécuter et «l'empêchement d'agir» tel qu'on l'entendra ici. Le fait de focaliser l'action peut être considéré comme le résultat de la volonté du sujet, ou comme une capacité qui provient de l'apprentissage d'une certaine activité. Certes le sujet doit faire certaines actions plutôt que d'autres. Mais il peut néanmoins disposer d'une marge de liberté pour modifier l'action, son déroulement, pour focaliser son attention sur une autre activité, dont il peut négocier les limites. En parlant d'empêchement d'agir, on s'intéresse à la manière dont les tâches sont élaborées, au fait qu'elles conduisent à des activités très restrictives, voire paralysantes, qui obligent les sujets à agir dans un univers particulièrement limité, avec peu ou pas de marge de manoeuvre. Dans ce cas, l'action du sujet est restreinte et définie dans des limites qui comportent peu ou aucune possibilité d'adaptation.

Ceci se produit déjà, d'une manière ou d'une autre, dans le propre processus de socialisation où l'apprentissage de la vie en société est également un processus. Cependant, bien qu'il y ait des règles de conduite, les possibilités d'agir sont innombrables et le sujet peut porter son attention sur des activités qui permettront l'usage de sa volonté et de son libre-arbitre. Néanmoins, plus on définit ce qu'il $\mathrm{y}$ a à faire de manière précise et restreinte, et plus on contraint, et empêche de faire quelque chose d'autre. Pour exécuter quelque chose de restreint, défini par certains types de tâches, la contrainte de ne pas faire d'autres choses devient un impératif. 
C'est particulièrement le cas dans les systèmes fortement taylorisés. Dans ce système d'idées, le " travailler » doit se constituer à partir de la réalisation de gestes précis, d'actions limitées au contexte de la tâche prescrite, en ayant pour but une optimisation des efforts destinés à la maximisation de la productivité, sans perdre de vue cependant la nécessité de préserver la force de travail.

Certes, on peut craindre qu'à trop centrer l'analyse sur le négatif, on en arrive à une vision très réduite de l'acte de travail. Ce n'est pas notre objectif. Il s'agit au contraire de centrer l'analyse sur l'effort pour ne pas bouger, l'effort pour ne pas penser, l'effort pour ne pas sentir. Ces efforts sont indispensables pour travailler dans des systèmes de production où les tâches fortement prescrites se résument à « répéter». On pourrait aussi se demander si cette approche ne se limite pas au travail à la chaîne, et à la vision qu'en a donnée Chaplin dans « les temps modernes », mais qui serait aujourd'hui dépassé, en voie de substitution par d'autres formes d'organisation du travail. Mais malheureusement, un nombre important de travailleurs, dans de nombreux secteurs de production, doivent travailler dans des situations qui exigent d'eux une activité qui fait partie du registre du « ne pas faire ». C'est d'ailleurs de plus en plus le cas dans le secteur des services : dans les centres d'appel, dans les banques ou dans la finance. Les «tâches de frappe », par exemple, sont de ce point de vue paradigmatique. Bien qu'elles soient parfois reléguées dans les souterrains invisibles de l'univers productif, sous-traitées, et externalisées, parfois même dissimulées dans la propre résidence de travailleurs, elles existent!

\section{3.- Méthodologie}

Les analyses ont été réalisées dans les centres d'appel de trois entreprises : une entreprise d'administration de cartes de crédit, une banque et une compagnie de téléphonie portable. Dans chacun des cas il y avait, de la part de la direction des entreprises, une demande initiale relative à des cas de TMS. Dans les deux premières entreprises, une Analyse Ergonomique de l'Activité a été réalisée et, parallèlement, une action inspirée de la méthode de la Psychodynamique du Travail. Dans le troisième cas, seule l'analyse proposée par l'ergonomie a été effectuée.

En premier lieu, des données ont été recueillies et des entretiens réalisés, dans les entreprises, auprès de représentants des ressources humaines, des secteurs commerciaux et d'opération directement responsables pour les centres d'appel. Les données relevées étaient relatives aux objectifs de l'entreprise et aux types de produits et services offerts aux clients. En ce qui concerne la production, des données relatives aux flux de production et à la division du travail dans les différents secteurs de l'entreprise ont été recueillies. On a également relevé le volume des appels reçus, les variations au long de la journée et les éventuelles influences de la saisonnalité. En ce qui concerne l'organisation du travail, des données relatives aux temps alloués, à la composition des équipes et à la division du travail au long des processus ont été relevées. Les données démographiques ont été travaillées en relation à l'ancienneté, l'âge, le sexe, et la scolarité. Il a également été relevé des données sur les arrêts maladie, les politiques d'embauche et de licenciement, et sur les politiques de formation de travailleurs.

L'analyse des tâches a été réalisée à partir de relevés relatifs aux procédés de travail, aux caractéristiques des postes de travail, à des données sur l'environnement, sur les outils et sur les équipements utilisés, y compris les logiciels. Il a ainsi été possible de mettre en relief le contenu des tâches et la relation de celui-ci avec l'organisation du travail.

En plus des relevés des données et des entretiens mentionnés auparavant, il a été créé, dans chaque entreprise, un groupe de travailleurs, dénommé "groupe opérationnel » qui a accompagné les travaux de l'équipe d'ergonomes au cours des études. Ces groupes étaient composés de travailleurs des centres d'appel qui se sont proposés comme volontaires, après une présentation initiale lorsqu'il leur a été montré une ébauche des études proposées ainsi que leurs objectifs. Ces groupes se réunissaient avec une fréquence hebdomadaire pour débattre de divers aspects du travail, et participaient également aux analyses des activités. Pour permettre la réalisation des analyses des activités, il a été effec- 
tué des observations directes, des photographies, des enregistrements audio et des films vidéo. Ces données ont été présentées et débattues durant les réunions du groupe opérationnel. Dans le cas de l'entreprise de cartes de crédit, des groupes de deux travailleurs ont été formés où l'un accompagnait le travail de l'autre (par l'écran de l'ordinateur et par l'écoute de l'appel) et où étaient soulignées et discutées les stratégies de navigation dans le système informatique et celles des réponses aux appels. En plus de ces groupes opérationnels, des ateliers réunissant la majorité des travailleurs des centres d'appel ont été réalisés pour présenter certains concepts de l'ergonomie, pour discuter les postes de travail et les postures ainsi que les problèmes posés par la tâche ? Dans le cas de l'entreprise de téléphonie, les groupes d'opérateurs n'ont pas été constitués, mais des observations directes, des films et des discussions avec les travailleurs volontaires ont eu lieu. Un rapport final a été élaboré, qui comprenait les données relatives aux études effectuées et les propositions d'amélioration. Ces résultats ont été discutés avec les participants du «groupe opérationnel » avant la présentation aux directions des entreprises.

En relation à l'autre partie des études - l'action basée sur les propositions de la psychodynamique du travail - il a été créé dans les entreprises des groupes de travailleurs qui avaient déjà été mis en congé maladie pour des problèmes ostéo-musculaires (TMS) et qui avaient réintégré les entreprises dans les mêmes secteurs ou dans des secteurs différents. Dans un premier temps, ces travailleurs ont été invités à une réunion dans laquelle la démarche a été présentée, et ceux qui étaient intéressés ont été invités à participer aux autres réunions (au nombre de 6 à 8 selon les études). Pendant les réunions, il leur était demandé de raconter leurs expériences du travail et à la manière dont ils étaient tombés malades. Les résultats de chaque réunion étaient donnés au groupe à la réunion suivante, et une discussion finale a eu lieu à la dernière réunion. Les résultats ont été, en partie, présentés aux directions des entreprises, avec l'autorisation des participants. Cette partie du travail n'a pas été effectuée dans l'entreprise de téléphonie.

\section{4.- Présentation des situations étudiées et résultats}

La demande initiale formulée par les professionnels des Ressources Humaines était liée à une augmentation significative des cas de congés maladies dues à des problèmes de TMS. Dans chacune des entreprises, plus de $10 \%$ de la population était en arrêt de travail. Dans la majorité des cas, il s'agissait de travailleurs jeunes et qui avaient peu d'années d'ancienneté. Outre la question de la santé des travailleurs, on pouvait observer des conséquences significatives en relation à la production puisqu'une quantité réduite de travailleurs devait prendre en charge la totalité des appels (aucune embauche n'ayant été faite pour remplacer les travailleurs en congé maladie, même temporairement). Des impacts sur la productivité se faisaient donc sentir, entraînant à leur tour les problèmes de qualité des services. Il existait en plus de cela une expansion de la demande de ces services qui restait sans réponse, faute d'une augmentation du nombre des travailleurs, aussi bien que d'une augmentation des ressources techniques. L'impact sur les travailleurs qui continuaient à travailler était significatif puisqu'ils subissaient une plus grande pression : devant prendre en charge le travail des absents, ils augmentaient le risque de tomber eux-mêmes malades.

Un fait intéressant vient s'ajouter à cette analyse. Cet univers de souffrance se produit dans des locaux de travail où les conditions matérielles pour la réalisation des tâches sont satisfaisantes en matière d'hygiène au travail. Il s'agit d'ambiances propres où existe une souffrance significative, une souffrance exprimée par des jeunes, contrairement aux maladies classiques du monde du travail dont l'expression apparaissait au bout de nombreuses années, en conséquence de l'exposition à un type quelconque d'agent nocif. Dans notre cas, nous nous trouvons face à une souffrance qui résulte de causes plus subtiles, invisibles au premier coup d'oeil. La contrainte imposée aux travailleurs provient de la manière dont le travail est conçu, de son contenu, de son organisation.

Les défis qui accompagnent la compréhension des causes de la souffrance sont significatifs. Un chemin alternatif pourrait être suivi à partir d'une notion dialectique entre le « faire » et le « ne pas 
faire ».

Pour produire les résultats escomptés, l'individu doit focaliser totalement son attention sur ce « faire », sur cet acte restreint au procédé défini. L'efficience des systèmes dépend de la répétition, dans le temps le plus court possible, des actions de service proposées. Pour « faire », pour se restreindre à cet acte, l'individu est obligé de « ne pas faire ». Plus les cadences sont rapides, plus les temps morts sont réprimés, et plus l'individu doit « ne pas faire » pour « faire ».

Les types de travail étudiés présentent un autre aspect intrigant, c'est que le service au client est un travail relationnel. Il se construit, entre le travailleur et le client, une relation définie par le contrat de service et délimitée dans l'univers de la tâche ou des règles établies. L'activité du travailleur est adressée à quelqu'un, et l'acte de service est le résultat d'une construction dialogique exécutée par le travailleur, en simultanéité avec les actions du client. Malgré la tentative, qui existe dans les entreprises étudiées, de conformer cette relation à la rationalité technique, cette relation pourrait être considérée comme le résultat du rapport entre trois rationalités - technique, du travailleur et du client. Ce qui existerait d'hégémonie dans la rationalité technique résiderait dans le fait que les actions de service effectuées par le travailleur et les actions de sollicitation faites par le client sont définies dans un cadre permis par une rationalité définie en amont, mais qui ne considère pas les difficultés et les aléas de la production de l'acte de service. Pour les travailleurs, le besoin de se maintenir dans le cadre de cette rationalité définit leur « agir » et, également, leur « ne pas agir ».

La complexité de l'acte de «travailler» dans ces conditions ne résiderait pas seulement dans le « faire », mais surtout dans tout ce qui doit être réprimé et le processus d'incorporation d'une autodiscipline et pour « ne pas faire ».

Certes, l'existence d'un cadre bien défini pour agir pourrait être considéré comme une référence sûre permettant aux travailleurs d'accomplir leurs tâches sans qu'ils aient besoin de se questionner quant au résultat de l'effort fait pour travailler. Cependant, de nombreux facteurs peuvent produire des confusions. Les critères établis en relation à ce qui peut et à ce qui ne peut pas être fait ne sont pas précis. Ce qui peut être considéré comme étant satisfaisant ou de qualité, à un moment donné, peut être différent à un autre moment. Ce qui est encouragé par un supérieur hiérarchique peut être réprimé ou même puni par un autre. Bien qu'elle soit préconisée comme une des maximes des systèmes de production inspirés de Taylor, la précision des critères n'existe pas dans ce genre de travail. Cela pourrait être un autre facteur qui cause la souffrance, dans la mesure où, dans la plupart des cas, tout l'effort fait pour « faire » / « ne pas faire » n'est finalement pas satisfaisant, aussi bien pour soi que pour le client.

D'autre part, la contrainte pour « ne pas faire » serait considérée comme un effet secondaire qui n'est pas recherché dans la manière de concevoir le travail. Mais ce n'est pas exactement cela que les enquêtes ont révélé. Le paradigme de la simplification (Morin, 1998) est celui qui s'impose durant la conception. Ce que les départements d'ingénierie et de méthodes recherchent, c'est de rendre le travail le plus simple possible, passible d'être défini par des règles et des procédés précis. De cette manière, les résultats obtenus dans les offres de service au client seraient plus sûrs dans la mesure où il est possible de définir, a priori, ce que l'on en attend.

Malgré la légitimité de la recherche de fiabilité dans le service, le paradigme de la simplification de la tâche est faux. La question qui se pose, en fonction des résultats des enquêtes, est la suivante : en plus du fait que dans l'exécution de la tâche il y aura toujours une quantité significative d'événements non prévus, qui modifient la tâche, le sujet se voit obligé de restreindre son action. Quel effort doit être produit pour que le travailleur arrive à construire le « ne pas faire »? Nous pourrions considérer comme un exemple de ce « ne pas faire » la situation, fréquente, où le travailleur sait ce qu'il pourrait faire pour résoudre le problème du client mais il ne peut pas le faire puisqu'il n'y est pas autorisé.

Dans la mesure où la personne n'est pas autorisée à faire quelque chose, il est nécessaire qu'elle lutte contre son impulsion de faire cette chose, il est nécessaire qu'elle la freine, qu'elle la réprime. La répression du « faire » est associée à la répression de la pensée et à la répression du désir. Réprimer 
la pensée devient une nécessité pour que la personne puisse se concentrer uniquement sur ce qui est prévu dans la tâche. Tout autre pensée doit être réprimée puisqu'elle peut influencer la performance individuelle et compromettre le « résultat du travail ». Pour ce qui est de la répression du désir, nous pouvons faire référence à l'impossibilité pour la personne de « bien faire » ce qu'elle est en train de faire : pour résoudre le problème du client, il ne faut pas être obligé de s'en tenir au procédé imposé. Nous pouvons encore ajouter la création de stratégies pour éviter de souffrir de la conscience de ne pas offrir un bon service, ou encore de ne pas pouvoir réagir aux agressions.

Pour travailler dans les situations étudiées, le travailleur se voit obligé de restreindre sa liberté de mouvements. Pendant plusieurs heures (journée de 6 heures) il doit rester assis, souvent à des postes de travail inadéquats. Rester longtemps assis, cela signifie empêcher un fonctionnement plus harmonique du corps, l'immobilité posturale étant contraire à la physiologie dans la mesure où elle rend la circulation difficile. En outre, rester à son poste de travail signifie restreindre la possibilité de satisfaire des nécessités physiologiques comme uriner, excréter, s'alimenter ou boire. Pour travailler, le corps doit apprendre à ne pas fonctionner ou plutôt à fonctionner d'une manière restreinte. Le coût de cet effort serait l'inconfort, les douleurs, les troubles, les lésions. Il est intéressant d'observer que pour exécuter des mouvements répétitifs, comme saisir des données, il est nécessaire d'immobiliser le reste du corps, puisque le mouvement se limite aux doigts et il est également nécessaire de maintenir la tête immobile pour arriver à focaliser l'attention sur la source principale d'information, c'est-à-dire, l'écran de l'ordinateur.

Le mouvement est restreint et « l'agir » aussi. Comme il a été dit antérieurement, ce que le travailleur peut faire pour le client, se trouve réduit à la discrétion qui lui est attribuée en fonction de la manière dont le travail est organisé. Dans la mesure où l'action dépend de procédures cognitives, en passant par les compréhensions des demandes des clients, les possibilités d'offrir un service, par les manières de procéder et par l'expérience personnelle du travailleur, le « faire » est un acte pensé dans un certain contexte. Si ce contexte est très limité, défini par des procédures rigides, le travailleur se voit contraint à ajuster sa pensée à ce qui peut être fait. Ce fait peut paraître étrange, les gens étant en principe libres de penser comme ils le veulent. Cependant, il n'en est pas exactement ainsi. Le «ne pas pouvoir faire » peut conduire à un «ne pas penser à » car réfléchir à quelque chose que nous faisons en sachant que nous ne pouvons pas le faire comme nous le voudrions, serait une cause de souffrance.

Penser à ce qui devrait être fait pour faire mieux, penser à d'autres manières de faire, échanger des idées avec des collègues pour améliorer le travail, toutes ces possibilités sont exclues de cet univers de production. Focaliser la pensée sur l'opération sur ce qui est prévu dans le cadre de la tâche devient alors stratégique pour éviter une plus grande souffrance. Paradoxalement, l'effort cognitif est composé de ce qui peut et doit être fait, de manière cadencée, répétitive, rapide, et également de tout ce qui ne doit pas être pensé parce que cela pourrait provoquer des erreurs et augmenter la souffrance. Le « ne pas pouvoir faire » pourrait avoir comme conséquence le « ne pas pouvoir penser ».

Un autre aspect paradoxal serait lié à la question de l'être, de l'existence du sujet au travail. À quel point la nécessité de maintenir un type de comportement conditionné à des paramètres restreints peut-elle créer une relation de dissonance avec l'être ? Il est possible que s'installe un conflit entre d'une part le "vouloir servir », le "vouloir faire » de la manière la plus adéquate étant donné ses caractéristiques personnelles et les caractéristiques du client et d'autre part les restrictions imposées par la conception du travail.

Les restrictions aux possibilités d'action sont déjà rencontrées dans les définitions des procédures opérationnelles. Pour éviter par exemple des confusions, il est demandé à la personne d'adopter un pseudonyme qu'elle utilise pendant son travail : elle répond au téléphone la journée entière en utilisant un nom qui n'est pas le sien. Même si elle y consent de bon gré, cela ne conduirait-il pas à un renfort de la distinction entre l'être au travail et l'être dans sa vie personnelle ? Quel type de dissonance serait ainsi construite entre l'acteur au travail et l'acteur dans la vie personnelle? Son identité serait-elle imprégnée par cette nouvelle « identité » assumée durant le travail ? 
Cette question serait-elle fausse ? Ce que nous sommes, c'est ce que nous faisons, ce sont les attitudes que nous adoptons, les actions qui nous sont permises. Dans ce sens, le travail est un modulateur de l'identité du sujet. Or, il s'agit d'un problème dans le cadre d'un travail trop limité. Les comportements à adopter les possibilités d'agir et de réagir le plus conformément possible avec les sentiments personnels sont réduits à un minimum. Le comportement escompté, la manière de parler au téléphone, les mots qui doivent être dits, ce qui ne peut pas être dit, tout ceci établit un cadre qui oblige le sujet à être lui-même le moins possible, pour que le service offert soit conforme à l'attente de l'entreprise.

Ce faire semblant pourrait être considéré comme un processus de dépersonnalisation, de réduction de l'existence du sujet au minimum pour rendre celui-ci productif. Ce ne sont pas seulement le geste et la cognition qui doivent êtres conditionnés et focalisés sur des tâches restreintes. L'être, lui aussi, ferait partie de la tâche, l'être, lui aussi, deviendrait, en grande partie, " prescrit ».

Ce fait n'est pas une nouveauté dans le monde des services, ni dans les entreprises étudiées. Ce processus serait une des tendances qui résultent du système d'idées issues de Taylor. Ce qui différencie considérablement la situation des services, c'est la rencontre avec le client. Le travailleur se trouve face à face avec le client, il est en contact avec le client par le téléphone. Pour se conditionner à une tâche restreinte, pour pouvoir faire partie du monde prescrit, la règle du jeu devrait également inclure le conditionnement du client ; il faudrait également prescrire, dans la mesure du possible, le comportement de celui-ci. La relation travailleur-client doit être conditionnée le plus possible à l'univers de la prescription, une relation réifiée finit par devenir un objectif, car il est nécessaire d'éviter les dérapages qui pourraient compromettre la productivité de celui qui opère.

En outre, toute possibilité de mauvais traitements, d'agressions verbales de la part du client doit être intériorisé, rapidement digéré, car une quantité de personnes attendent dans la queue, anxieuses pour qu'on s'occupe d'elles et de leurs nécessités.

Néanmoins, maintenir le client dans ce cadre restreint n'est pas toujours facile. Nous avons choisi un exemple dans lequel il est possible de constater que la rigidité du système peut entraîner des situations difficiles pour les opérateurs.

Opérateur (Op) - La Banque, centre d'appel, Silvia, bonjour.

Client (C) - Bonjour, Silvia. S'il vous plaît, vérification de compte bancaire.

$\mathrm{Op}$ - S'il vous plaît, votre agence.

$\mathrm{C}-$ Trois zero huit six.

Op - Compte?

$\mathrm{C}-$ Un trois neuf quatre neuf deux.

Op - Code d'accès?

$\mathrm{C}$ - A comme Arnault, B comme Bach vingt-six.

Op - Un moment, s'il vous plaît... M. R, quelle opération vous désirez ?

$\mathrm{C}$ - Je voudrais savoir mon solde.

$\mathrm{Op}-$ Quatre cent quatre-vingt-six reais et soixante centimes.

$\mathrm{C}-\mathrm{Ah}$, d'accord. Quatre cents et...?

$\mathrm{Op}$ - Quatre huit six virgule six zéro.

$\mathrm{C}$ - Il a été encaissé un chèque de six cents soixante reais, c'est ça ?

Op - Un moment s'il vous plaît, je vais vérifier...Il a été encaissé le six novembre.

$\mathrm{C}$ - Le six novembre ? Ok. Je voudrais transférer quatre cents...

L'opérateur interrompt.

Op - Quel est le transfert que vous voulez effectuer, pour un compte bancaire ou un compte épargne?

C - Je voudrais transférer d'un compte bancaire...

L'opérateur interrompt.

$\mathrm{Op}$ - S'il vous plaît, quel est le numéro de l'agence et du compte à être crédité ?

$\mathrm{C}$ - L'agence à être créditée zéro quatre six six

$\mathrm{Op}$ - Le numéro de compte ?

$\mathrm{C}$ - deux sept deux quatre zéro trois. 
Op - Le montant?

$\mathrm{C}$ - quatre cent cinquante reais.

Op — Le débit c'est sur le premier compte que vous m'avez informé ?

$\mathrm{C}$ - Comment?

Op — Le débit...

Le client interrompt.

$\mathrm{C}$ - Oui, certainement.

Op - Pour confirmer, le transfert compte bancaire débité agence trois zéro huit six, compte un trois neuf quatre neuf deux, valeur quatre cents cinquante reais, compte bancaire crédité agence zéro quatre six six, compte deux sept deux quatre zéro trois, au nom de Mr. X, vous le confirmez?

$\mathrm{C}$ - Oui.

Op - Transfert effectué.

$\mathrm{C}$ - Ok. Maintenant, eh..., agence trois mille.

Op - Oui.

$\mathrm{C}$ - Compte bancaire quatre neuf cinq zéro neuf sept.

Op - Quelle information désirez vous ?

C — D'abord, le solde.

Op - Solde partiel débiteur de vingt-quatre reais quatre-vingt-dix centimes.

$\mathrm{C}$ - Ok. Est-ce qu'il a été crédité un chèque de...

L'opérateur interrompt.

Op - Un moment, je vais vérifier.

$\mathrm{C}$ - Silvia.

Op - Je vous écoute.

C - Attendez que je finisse de parler, s'il vous plaît. Je veux savoir s'il a été crédité cinq cents reais sur mon compte.

Op - Vous voulez savoir s'il y a eu un crédit...

Le client interrompt.

$\mathrm{C}$ - S'il y a eu un crédit de cinq cents reais sur mon compte et...

L'opérateur interrompt.

Op - Un moment, s'il vous plaît...

Le client interrompt.

$\mathrm{C}$ - ...entre avant-hier et aujourd'hui... et vous attendez que je finisse de parler, s'il vous plaît !

$\mathrm{Op}$ - Le six il y a eu un transfert à crédit d'une valeur de cinq cents reais.

$\mathrm{C}$ - Ok. Je voudrais un transfert de ce compte zéro quatre six six...

L'opérateur interrompt.

Op - Vous allez effectuer un autre transfert ?

C - C'est ça!

Op - C'est pour débiter sur ce compte?

$\mathrm{C}$ - Correct.

Op - C'est pour un compte bancaire ou un compte épargne?

$\mathrm{C}$ - Agence zéro quatre six six...

L'opérateur interrompt.

Op - Le transfert, c'est pour un compte bancaire ou un compte épargne ?

Le client monte la hauteur de la voix.

C - C'est pour un compte bancaire, Silvia!

Op - Numéro de l'agence?

$\mathrm{C}$ - Zéro quatre six six.

Op - Compte?

$\mathrm{C}$ - Deux sept deux quatre zéro trois.

Op - Quel est le montant?

$\mathrm{C}-\mathrm{Eh}$.... trois cent cinquante reais.

Op - Pour confirmer, le transfert compte bancaire débité agence trois zéro zéro zéro, compte quatre neuf cinq zéro neuf sept, valeur trois cents cinquante reais, compte bancaire crédité agence zéro quatre six six, compte deux sept deux quatre zéro trois, au nom de Mr. X, vous le confirmez ? $\mathrm{C}$ - Je le confirme.

Op — Transfert effectué. 
C - Silvia, s'il vous plaît, quel est le nom de votre supérieur?

Op - Il y en a plusieurs.

$\mathrm{C}$ - Ok. Dites-moi, au moins deux.

Op - Márcia...

C - Márcia...

Op — Celle-là c'est l'encadrement direct. Si vous voulez parlez avec elle...

C - Márcia.

Op - C'est ça. Voulez-vous parler avec elle ? Un moment je vous la passe.

$\mathrm{C}-\mathrm{S}$ 'il vous plaît.

Tableau 1 - Extrait d'un dialogue entre opérateur et client.

Table 1 - Extract of a dialogue between operator and client.

Avec cet extrait, nous pouvons observer que le client ne suit pas la logique du système technique, vu que son comportement ne suit pas la procédure. À plusieurs reprises, l'opérateur doit interrompre le client et lui poser des questions selon la séquence imposée par le logiciel, centrée sur la logique technique, base du travail des concepteurs. Le client de son côté suit plutôt une logique de raccourcis. Il essaye d'aller vite, il veut les informations tout de suite. Mais le système informatisé l'empêche, et alors il devient agressif et menaçant. Comment se retrouve l'opérateur (opératrice) dans cette situation? Il est obligé de suivre la procédure, mais il est confronté avec des questions qui sont posées autrement par le client. En plus, le travailleur connaît les problèmes liés aux défaillances du système technique, qui l'obligent à procurer des excuses face aux clients qui de leur part ont parfois des demandes qui ne peuvent pas être satisfaites. Soulignons que la logique des clients ne suit pas la linéarité prévue lors de la conception du système de production.

Pour les travailleurs qui, dans les cas étudiés, étaient en majorité jeunes du sexe féminin, ce processus est vécu comme étant très douloureux : la restriction imposée à l'être atteindrait directement quelque chose qui est très important dans la jeunesse, c'est-à-dire les rêves, l'espérance, ce que l'on veut. Dans ce sens, le risque de tomber malade serait significatif, le corps contraint à peu de mobilisation, le traitement incessant d'informations, l'agression intériorisée, la restriction à la liberté de penser et de vouloir, pourraient, comme cela a été constaté pendant les séances de discussion réalisées pendant les études, résulter en une souffrance significative. Ce scénario serait propice à l'apparition de troubles comme les TMS et autres types de problèmes de santé, tels que troubles de la parole ainsi que des différentes expressions de souffrance psychique. Parmi les résultats des études développées par notre équipe, les arrêts maladie liés aux TMS atteignaient 10 à $14 \%$ de la population étudiée. L'incidence et la prévalence d'autres problèmes de santé étaient moins significatives, toutefois il y avait des signes préoccupants. Il y avait des diagnostics de problèmes liés aux cordes vocales, ainsi que des symptômes référés (perte de voix). Il n'y avait pratiquement pas d'arrêts maladies dus à des troubles psychiques. Toutefois les services de santé avaient déjà signalé une augmentation significative de la consommation des médicaments psycho actifs ainsi que des plaintes liées à la souffrance psychique.

Ces problèmes de santé seraient-ils de véritables maladies de l'empêchement?

Lorsqu'il tape sur le clavier de l'ordinateur, le travailleur maintient pratiquement immobile tout son corps et exécute des mouvements exclusivement avec les doigts ; il est pratiquement impossible de maintenir une activité de saisie de données sans adopter une posture fixe. Dans ce cas, de grands segments corporels sont maintenus statiques pour permettre un mouvement précis des doigts. Ce fait ne se restreint pas à ce type de tâche : tout travail de précision exige en effet de tels maintiens de posture, ce qui fait entrer en jeu les questions du temps durant lequel la posture est maintenue, et l'existence d'appui divers pour les masses corporelles. Il faut également remarquer que plus le rythme de production est poussé, plus la posture sera rigide, en fonction d'une contracture musculaire croissante, principalement de la musculature dorsale.

En ce qui concerne le temps, maintenir une posture statique parce que la tâche l'exige peut ne pas 
être problématique si cela se produit durant des périodes courtes. En effet, dans ce cas, la posture ne se rigidifierait pas puisqu'il y aurait alternance de postures. Mais dans les cas étudiés la posture était maintenue pendant des périodes longues, et la marge de variations au long de la journée de travail était étroite. Cela était dû au fait que pratiquement toute l'activité se réalisait face à un ordinateur. Les variations possibles de la posture étaient très réduites, elles se limitaient à de petits mouvements de hanche, de jambes, à quelques changements de position de la colonne vertébrale. Par contre, les épaules, la partie cervicale de la colonne, les bras et les avant-bras restent pratiquement immobiles.

De nombreux éléments contribuent donc à attribuer les difficultés à un «empêchement» dans la mesure où ce que la tâche exige et les possibilités offertes pour son exécution sont très strictes, se résumant parfois à une seule. Le mouvement est extrêmement réduit et restreint. Alors qu'une petite partie du corps produit un mouvement constant et limité, le reste du corps reste immobile. Le corps est, pour une bonne partie de la journée de travail, empêché de se mouvoir.

\section{5.- Les facteurs de l'empêchement}

Dans cette section, nous avons sélectionné et regroupé des données qui nous aident à montrer et à discuter le thème de l'empêchement, ainsi que sa relation probable avec la genèse des TMS. Il ne s'agit pas de fournir une vision monolithique, stéréotypée. Au contraire, il s'agit de montrer, dans la singularité des situations, les facteurs qui sont à l'origine du « ne pas faire », de l'empêchement. Pour en faciliter l'entendement, nous avons séparé les aspects physiques, cognitifs et psychiques.

\section{1.- Le mouvement soumis à l'empêchement}

Le travailleur ne pouvait pas se lever, il devait rester à son poste de travail durant des périodes prolongées. Dans les cas des centres d'appel étudiés, il y avait des situations où les travailleurs devaient rester assis, car il leur était interdit de répondre aux appels debout en alternant les postures. La journée de travail était de 6 heures, avec un intervalle de 15 minutes pour le repas et pour aller aux toilettes.

L'inadéquation des postes de travail et des équipements constituait un aspect aggravant de la situation. L'adoption d'une posture plus confortable était le plus souvent pratiquement impossible puisqu'il n'y avait pas d'ajustements suffisants pour régler la hauteur des sièges ni des plans de travail. En outre, l'espace du poste de travail, principalement celui du plan de travail, était très souvent exigu, peu d'espace restant donc en dehors des équipements et des outils de travail. Et, comme il n'était pas possible d'appuyer les coudes sur le plan de travail, les travailleurs utilisaient les bras des fauteuils (quand ils avaient des fauteuils au lieu d'avoir des chaises) ou gardaient les bras en suspens, utilisant la musculature des épaules pour maintenir la posture exigée. Dans certains cas, en fonction de l'inadéquation des équipements, les travailleurs maintenaient en extension l'un des bras, ou les deux.

Des postures plus confortables ne sont pas possibles en fonction de l'exiguïté des postes de travail et du besoin de manipuler certains équipements comme l'ordinateur (clavier et souris) et les équipements pour la communication téléphonique. La situation des caissiers de banque et des clavistes ressemblait beaucoup à celle que nous venons de décrire : la durée de la journée de travail était la même, les postes de travail étaient inadéquats, et les équipements exigeaient que les membres supérieurs soient maintenus dans des postures inconfortables. En outre, dans toutes les situations, il existait une stricte exigence de résultats, le rendement de chacun étant contrôlé, en temps réel, soit par des systèmes informatisés, soit par une supervision directe.

Ainsi, avec Bouisset (1992), nous soulignons que les mouvements qui sont nécessaires à l'exécution du travail tout en étant limités à un segment restreint du corps - les mains - exigent un non-mouvement du reste du corps. De cette manière, nous pouvons penser au phénomène de l'inflammation et de la douleur comme étant le résultat de deux situations apparemment opposées, celle du mouvement 
excessif concomitamment à celle de l'absence de mouvement. Les mouvements des doigts joints à des postures inconfortables comme l'extension des poignets pourraient, à eux seuls, causer des troubles ou des lésions, en fonction de la friction entre tendons et gaines, par exemple. D'un autre côté, la posture statique peut, en fonction de différents degrés de contraction musculaire, être responsable d'une réduction de l'apport de sang dans diverses parties des membres supérieurs, ainsi que de pincements de structures nerveuses situées près de la partie cervicale de la colonne vertébrale ou en tout autre endroit des membres supérieurs. Encore, la propre compression mécanique provoquée par l'appui de parties molles de l'avant-bras peut être un facteur causal de lésions.

Nous avons choisi un extrait d'une observation du travail dans un centre d'appel. La chronique d'activité issue de l'observation est montrée dans la figure ci-dessous (Figure 1). Il s'agit d'une entreprise d'opération de cartes de crédit. L'appel en question a une durée de 5 minutes et 20 secondes. Pendant cette période, l'opérateur reste assis et il n'y a pas de mouvements du tronc. Essentiellement, les mouvements se limitent aux bras et mains, ainsi que les changements de regard et quelques mouvements de la tête.

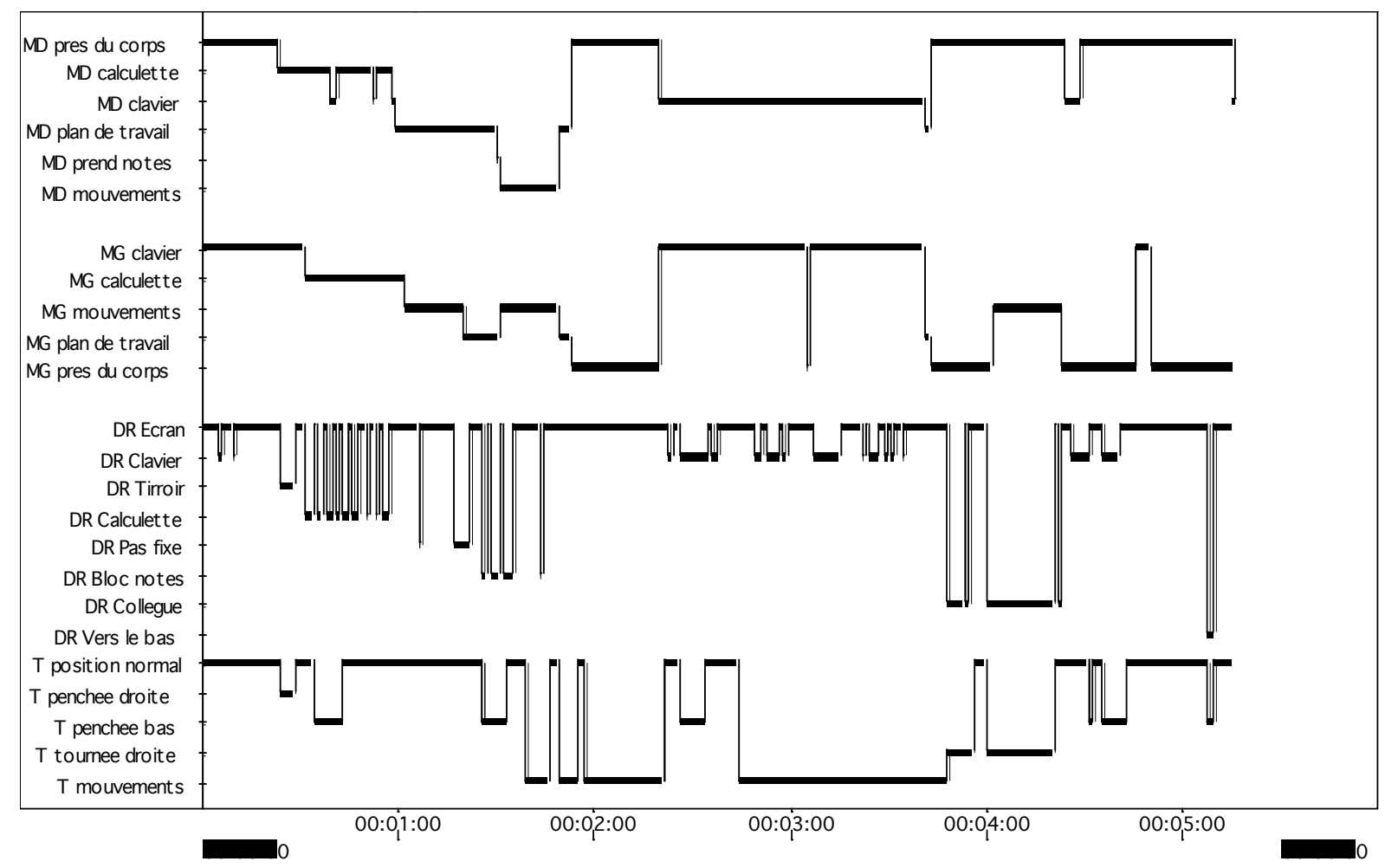

$\mathrm{MD}$ : main droite ; $\mathrm{MG}$ : main gauche ; $\mathrm{DR}$ : direction du regard ; $\mathrm{T}$ : tête $M D$-write hand ; $G$-left hand; DR-look direction; $T$ - head

Figure 1 - Extrait d'une observation dans un centre d'appel.

Figure 1 - Extract of an observation in a call center.

Les mouvements des bras sont assez courts. Ils se déplacent vers le clavier, lorsque l'opérateur saisit, quand il se sert de la calculette et quand il prend quelques notes sur une feuille « pense-bête ».

La chronique d'activité montre que les mouvements concernant les bras et les mains sont moins nombreux que ceux des changements du regard. En effet, il est possible de vérifier que pendant certains moments les changements de direction du regard s'intensifient. Par exemple, lorsque l'opérateur utilise la calculette, son regard s'alterne entre la calculette et l'écran. Il y a aussi des moments où l'opérateur cherche des informations sur le système informatisé et son regard est dirigé vers l'écran et le clavier. En ces moments, on observe aussi des mouvements des mains et doigts car l'opérateur se 
sert du clavier pour saisir des données ou pour passer d'une page à l'autre du système informatique. Par ailleurs, lorsqu'il prend des notes ou lorsqu'il consulte son collègue à côté, il y a également des changements de la direction du regard. Dans ce cas, au fur et à mesure qu'il se renseigne auprès de son collègue, il l'écoute et regarde sur l'écran. En dehors de ces mouvements des membres supérieurs, l'opérateur reste quasiment immobile sur sa chaise, à l'exception de quelques mouvements de la tête.

\section{2.- L'empêchement physiologique}

Dans de nombreuses situations, compte tenu de la manière dont le travail était organisé, il n'était pas permis aux travailleurs de satisfaire leurs nécessités physiologiques au moment nécessaire. Dans le cas des opérateurs de centres d'appel étudiés, il y avait des horaires prédéfinis pour aller aux toilettes. Dans le cas les plus urgents ils devaient consulter la supervision pour obtenir une autorisation, cependant des témoignages de travailleurs montrent qu'ils se sentaient contraints de ne pas sortir en dehors des horaires pré-établis par peur de sanctions de la part des chefs.

À ce propos, Guyton et Hall (1997) soulignent que les troubles du système urinaire, particulièrement la cystite chez la femme (des symptômes brûlants lors de la miction, des douleurs à l'urètre ou à la vulve, des crampes plus ou moins violentes dans le bas-ventre) sont très fréquents à cause de la rétention d'urine dans la vessie. Retenir l'urine serait aussi empêcher le fonctionnement du corps, vu que le désir conscient d'uriner est précédé d'un réflexe nerveux mais, qui peut être inhibé par les centres corticaux. La question est de savoir quelles sont les limites acceptables pour une telle rétention, qui ne peuvent pas être mis sur procédure, mais qui devraient être sur commande du sujet et non de l'entreprise.

\section{3.- L'action soumise à l'empêchement}

Dans diverses situations analysées, les travailleurs ne pouvaient pas agir pour résoudre certains types de problème, même lorsqu'ils possédaient la connaissance et le savoir-faire qui le leur aurait permis. Le mode de division des procédés de production et de définition des stratégies des entreprises empêchaient la réalisation d'actions utiles dans la quête des bons résultats. Nous considérons ici également les cas où il n'était pas permis de passer aux clients d'autres informations que celles qui étaient prévues. Ces faits se produisaient principalement dans les cas de réponse aux appels de clients, où, en fonction du découpage effectué par les entreprises, il n'était pas permis à ces travailleurs de résoudre les problèmes des clients ni même de donner des informations utiles pour résoudre le problème quand cela ne faisait pas partie du cadre prédéfini.

\section{4.- L'être soumis à l'empêchement}

Des situations de réponse aux appels des clients, du genre de celles que nous avons rencontrées dans les centres d'appel, constituaient des limitations considérables au processus d'identification avec le travail. Outre le conflit relatif à la possibilité de « faire », réalisant ce qui, en principe, serait l'objectif de leur travail - servir le client dans certaines limites - il y avait, dans la définition du contenu de la tâche, des aspects significatifs qui empêchaient un processus d'identification avec le travail. Nous pouvons citer ici le changement de nom et l'adoption d'un procédé de communication où le ton de voix, le type de phrases et ce qui peut ou non être dit sont prédéterminés. Les travailleurs, pour réaliser ces tâches, se transforment en personnages distincts d'eux-mêmes. Comme il l'a été dit antérieurement, dans de nombreuses situations, les travailleurs se voyaient obligés de ne pas faire certaines choses, même si cela allait contre leur désir et contre ce qui leur paraissait en accord avec ce qui serait adéquat en tant que réalisation de leur travail. Les affirmations des participants des groupes montrent que la distance en relation à l'image de soi, comme le fait d'être « différent » au travail et hors du travail, peut être considérée comme un signe de cette difficulté d'identification avec leur activité. L'un 
des exemples les plus significatifs est l'expérience racontée par les travailleurs concernant les doutes liées à leur propre identité, une fois que, en contact avec le client, ils sont obligés de dissimuler. Ils se demandent $\mathrm{s}^{\mathrm{c}}$ ils dissimulent tout le temps, même avec leurs amis, leurs conjoints, leurs enfants.

\section{6.- Discussion}

La discussion sur la genèse des maladies connues comme les TMS est, en partie, basée sur la question du mouvement, de la vitesse, de la force et de la répétitivité. À partir de diverses connaissances issues de la biomécanique, il est possible d'expliquer pourquoi se produisent les processus inflammatoires des gaines, tendons et autres structures des articulations. Dans la majorité des cas, ces types de maladie sont diagnostiqués dans les membres supérieurs et sont fondamentalement en relation avec certains types de mouvement des doigts, des mains, des bras et des avant-bras. D'un autre côté nous pouvons également analyser la genèse de ces problèmes sous l'aspect de l'absence de mouvement, de l'immobilité de certaines parties du corps, du processus qui fait que se rigidifie la posture. Les activités décrites avant, ainsi que de nombreuses autres, exigent du travailleur qu'il maintienne une posture définie pour pouvoir opérer.

En outre la question du " travailler » en situation de service pourrait être analysée par rapport aux conséquences dues à l'impossibilité de faire ainsi qu'aux conséquences liées aux blocages du rapport interpersonnel et aux possibles agressions à l'individualité génératrices de conflit entre le besoin de « faire semblant tout le temps » et le désir de bien faire et de s'identifier avec son travail.

La définition de service contient une dimension subjective, ou mieux, intersubjective, puisqu'il y a toujours relation avec un autre sujet. Dans la mesure où la tâche empêche le sujet d'effectuer l'acte de service conformément à la nécessité du client, un conflit s'établit entre ce que celui-ci aimerait faire et ce qu'il est obligé de faire. Entre ce qui est nécessaire, désiré, et ce qui est réalisé se trouve un vide : l'acte soumis à l'empêchement. Cette situation serait contraire à ce que propose Zarifian en relation à ce que devrait être le travail das les services :

«Travailler, c'est engendrer un service, c'est-à-dire une modification dans l'état ou les conditions d'activité d'un autre humain ou d'une institution. (...) En effet, le meilleur moyen de parler des clients d'une façon concrète et réflexive est de considérer le produit comme un service rendu à des destinataires précis, par rapport à des usages clairement définis, à des problèmes et des applications concrets que ces clients peuvent faire du produit ainsi offert au sein de leurs propres activités » (Zarifian, 1999, p.45).

Nos études montrent qu'il y a conflit entre, d'un côté, la communication divulguée dans les entreprises à propos de la qualité des services effectués, où l'on rencontre des expressions telles que « offrir un bon service », « connaître les nécessités du client », " plaire à un client-roi et l'enchanter », « personnalisation », « segmentation », « fidélisation » et, de l'autre, la réalité que l'on rencontre. Traiter la qualité du service à partir du point de vue des sujets concernés serait une alternative. Ce point de vue est construit par la rencontre qui découle de l'offre de service, c'est-à-dire considérée comme le résultat d'une relation intersubjective réalisée par l'entremise d'une action qui a été à l'origine d'un certain type de modification pour le client. Le jugement en relation à la qualité du service est, en partie, construit par la relation de service qui a eu lieu et par la manière dont les différents acteurs - opérateur, client, collègues, superviseur - agissent au long du processus. En tant que destinataire de l'action, le client juge à quel point l'action a servi à répondre à ses attentes, à quel point cette interaction avec le travailleur comme représentant de l'entreprise l'a satisfait.

Aussi bien la modification de l'état du client que la satisfaction en relation à l'action et à son résultat peuvent être considérées dans les deux sens, puisque le travailleur lui-même se transforme à mesure qu'il offre le service. La question de la subjectivité, considérée dans la perspective de l'identification au travail, se pose dans la mesure où le travailleur se trouve empêché de construire cette relation avec le client. 
Lorsqu'il n'y a pas empêchement, le travailleur se "met lui-même » au service de l'autre, en ayant le but de faire quelque chose pour cet autre. Cette « utilisation » de soi-même est chargée de sens, puisque l'enjeu en est l'authenticité de l'acte. Durant tout ce procédé, qui est intersubjectif, le travailleur vit un processus contradictoire entre se mettre au service de l'autre, disposer de soi, et les empêchements créés par la tâche qu'il doit exécuter. La sensation d'étrangeté, identifiée par plusieurs travailleurs et exprimée dans les groupes de travail en relation à cet empêchement, pourrait être en partie imputée à la contradiction entre un acte authentique et le résultat d'une action soumise à l'empêchement.

Rabardel (2005) discute la question de la dialectique entre le sujet capable qui aurait la possibilité d'intervenir dans le monde, dans une dynamique de devenir et développement, en s'appuyant sur des processus de construction d'instruments subjectifs et son pouvoir d'agir. Selon lui, la perte du pouvoir d'agir, largement retrouvée dans les situations de travail étudiées dans cet article, empêcherait le développement des capacités des sujets. Le contenu et l'organisation du travail seraient des sources d'empêchement pour les médiations réflexives, importantes, selon l'auteur, pour le développement des sujets.

La question intersubjective peut également être analysée sous l'angle du plaisir trouvé dans la relation avec le client et du fait qu'éventuellement cette relation peut répondre aux attentes aussi bien de l'opérateur que du client, quand il est possible de « disposer de soi » de manière authentique.

En ce qui concerne les possibilités d'identification que les actes du «travailler » présentent, nous adoptons le concept de « technologie de soi » comme l'un des concepts qui peuvent faciliter ce débat. Contrairement à ce que proposait Morehouse (1958), quand il affirmait que dans l'analyse de la technologie toute référence à l'intériorité devait être éliminée, liant ainsi la technique au domaine exclusif des sciences de la nature et des sciences appliquées. La technologie, sous l'influence de l'anthropologie, commence a être considérée comme une science humaine des techniques, éliminant le concept qui considère la technologie comme synonyme des dispositifs machinaux. La technique, quelle qu'elle soit, exerce une incidence sur le réel en tant qu'acte situé dans une culture et fruit d'une histoire dans la société.

La technique est un acte traditionnel efficace (Mauss, 1934, apud Dejours, 1997), ce qui élimine l'exigence de la présence des dispositifs machinaux. L'acte technique suppose un maniement qui est propre au corps de celui qui agit. En outre la technologie ne se réfère pas aux objets techniques, mais à l'histoire des conduites et des habiletés humaines (Mauss, op. cit. ; Haudricourt, 1987 ; Bohle, \& Milkau, 1991). Ainsi surgit non seulement une critique de la rationalité du concept de technologie mais aussi le concept d'activité « subjectivante » (Bohle, \& Milkau, op. cit.), où l'exigence objective de l'activité et le processus subjectif s'équilibrent l'un l'autre. Intégrer cette approche de la technologie à l'acte qui inclut l'intériorité du sujet nous renvoie à une généalogie du sujet moderne (Dreyfus, \& Rabinow, 1983).

\section{7.- Conclusion}

Il nous semble possible de discuter que la souffrance exprimée par les TMS et aussi par d'autres troubles et maladies, pourrait être le fruit de l'empêchement produit par une organisation du travail très restrictive et par un pauvre contenu des tâches, ainsi que par des mécanismes de non reconnaissance du travail accompli.

Dans ce sens, nous rejoignons Clot (1995) lorsqu'il souligne que le taylorisme ne demande pas trop au travailleur, mais trop peu. Pour l'auteur, en choisissant le mouvement qui réclame de sa part le moins d'entremise, on prive l'homme de son initiative. Cela veut dire que, focalisé sur le geste, le taylorisme est simultanément sa dénégation et c'est ce geste à la fois prescrit et interdit qui est plus coûteux. 
Il est intéressant de signaler les questions posées par Clot et Fernandez (2005) à propos du geste professionnel. Selon eux, le geste répétitif est un geste empêché, contrarié, refoulé, et qu'un geste est répétitif chaque fois que le mouvement est amputé de ses possibilités de développement. Selon eux, il s'agirait d'une impossibilité rencontrée par les sujets à créer des contextes variés pour y exécuter les gestes professionnels.

En faisant appel à des concepts proposés par Vigotsky et discutés par Clot (1999), il nous semble que les émotions considérées comme des vrais moteurs de l'action sont réprimées, comme nos études l'ont montré. Les travailleurs de ces centres d'appel n'ont pas la possibilité d'exprimer leurs émotions, même pour les transformer en attitudes positives envers les clients et les collègues. Le fait de ne pas avoir un " espace public » pour discuter les questions liées à leur travail serait aussi un empêchement au développement du genre professionnel, ainsi que le style personnel tributaire du métier, des actions des sujets et des caractéristiques personnelles. Le métier ne peut pas se développer car les travailleurs n'ont pratiquement pas d'espace pour les échanges et restent peu de temps dans l'entreprise.

Est-ce que le fait de devoir se donner pendant des heures et soutenir une cadence qui ne peut pas être régulée par les travailleurs eux-mêmes ne provoque pas une situation semblable à celle décrite par Périlleux (1999), où il serait difficile pour les personnes soumises à des régimes de formalisme rigide de construire une cohérence bibliographique. Dans ce cas, nous serions face à un processus de décompensation psychique. N'y aurait-il pas une certaine similitude avec le cas des TMS et les autres problèmes de santé vécus par les travailleurs des centres d'appel étudiés ?

Il semble aussi que les situations étudiées pourraient se comparer à celles qui sont discutées par Jayet (1999), c'est-à-dire les entreprises marchent, mais les travailleurs souffrent car la dynamique des relations sociales de travail repose sur la violence, la peur, la rivalité et non sur la concertation. Les TMS seraient-ils ainsi produits?

En outre, il serait possible de discuter l'empêchement comme une source de répression de soi, dans un sens similaire à celui présenté par Pezé (2000). Elle discute le travail au féminin, en accentuant, entre autres questions la déqualification et la mise en échec de l'identité au travail. On pourrait, en s'inspirant de la perspective psychosomatique, expliquer au moins en partie la genèse des problèmes de santé retrouvés dans notre étude comme la faillite des possibilités de représentation, du débordement des capacités de liaison de la psyché, d'une vraie situation d'impasse pour le sujet.

En s'éloignant de cette perspective, Dejours (2001) propose la notion d'agir expressif. Il y aurait une dimension psychodynamique et expressive, en tenant en compte de l'existence d'adressage, une dramaturgie propre à l'agir. Dans cette perspective, des troubles, comme les TMS, seraient aussi le fruit d'une situation où l'empêchement résultant des choix organisationnels atteindrait aussi les processus imprescriptibles, hétéronomes à l'ordre des mécanismes et relevant de l'ingéniosité. Dejours (2000) parle encore, en se référant aux travaux répétitifs sous contrainte de temps, d'une sorte d'état de torpeur psychique, une espèce d'anesthésie de la conscience douloureuse de son état. Selon lui, il y aurait des désordres importants du fonctionnement psychique et de l'activité de la pensée. Peut-on alors faire référence à une conséquence de l'empêchement liée à l'identité des travailleurs, de leur psyché, de leur « être ».

Pourrait-on dire que le fait de ne pas pouvoir « faire » et ne pas pouvoir « être » serait un danger pour le sujet, dans la mesure où il y aurait encore moins de possibilités d'adressage et de construction de relations avec les collègues et clients ?

Dans la perspective du bien-être, telle que l'a proposée Maggi (2003), les situations de travail étudiées montrent que les choix organisationnels faits par ces entreprises, laissent très peu d'espace pour les sujets, même si la référence est «l'espace discrétionnaire ». Or, les sujets n'ont même pas l'autonomie pour décider le moment de s'arrêter, de changer les cadences. Pourrait-on parler des choix organisationnels qui réduisent ou même qui empêchent les sujets, réduisant les espaces discrétionnaires au respect des procédures? 
Le contrôle et le monitorage des travailleurs, grâce aux technologies informatiques, sont des instruments puissants pour construire une sorte d'empêchement structurel, où prévaut le non faire sur le faire. Le contrôle du travail par ces systèmes informatisés et l'application stricte d'une rationalité prescriptive est en conflit avec le besoin de plasticité inhérent aux activités de service, aux relations de service (Arbix, \& Sznelwar, 2002).

Ce type de travail serait plus qu'un obstacle au sujet (Sznelwar, \& Massetti, 2002). Il s'agirait plutôt d'obstacles qui ne peuvent pas être transposables : des vrais empêchements, des vraies sources de souffrance et de maladie.

Il nous semble donc nécessaire de changer le point de vue des entreprises. Les choix organisationnels intrinsèques au travail imposé aux travailleurs sont tributaires d'une vision qui repose sur un travail simple, dans lequel il suffirait de respecter les consignes et les procédures pour l'accomplir. A quoi arrive-t-on ? À un travail dépourvu de sens, à l'empêchement des possibilités de développement professionnel, à l'impossibilité de l'accomplissement de soi, à la maladie. Des alternatives existent. Il ne s'agit pas d'un travail simple, ni simplifiable. Il faut adopter des solutions, aider les entreprises à adopter des choix organisationnels qui visent le contraire de l'empêchement.

Proposer des alternatives concernant la manière de concevoir le travail dans les situations décrites auparavant est un défi pour l'ergonomie. Comment proposer des transformations qui aboutissent à la fois à des tâches intéressantes, ayant du sens, qui deviennent une base solide pour la construction de la santé des travailleurs et qui soient productives pour les entreprises ?

Pour développer un processus de re-conception nous pouvons faire appel aux propositions de Béguin (2005). Selon lui, un tel processus devrait se baser sur des stratégies en vue de faciliter les genèses professionnelles. Ceci étant, ce processus devrait aussi se baser sur une méthode dialogique fondée sur un apprentissage et une confrontation entre des formes de connaissances différentes construites à partir de mondes distincts. La question centrale serait de rendre possible un déplacement et une appropriation du monde de l'autre, des concepteurs et des usagers. Nous rejoignons encore le point de vue de l'auteur que le monde des opérateurs n'est ni visible, ni disponible. En plus, leur point de vue n'est pas considéré comme légitime, vu qu'ils ne sont pas considérés comme des contributeurs. Parfois, nous avons l'impression que leur travail n'est pas reconnu comme une action qui ajoute de la valeur au service, il s'agirait, vu par le point de vue de certains concepteurs, comme un mal nécessaire. Voilà des grands défis.

Un changement de paradigme serait donc nécessaire. Les processus de production conçus à partir de paradigmes fonctionnalistes, sont à la base des activités analysées. Ces activités sont la conséquence de tâches restrictives, dans lesquelles l'action prévue pour les opérateurs est délimitée de manière très étroite. Les résultats des actions des travailleurs ne sont pas toujours productifs, c'est le cas, par exemple, des problèmes qui ne sont pas résolus par les opérateurs et qui, finalement sont résolus par la hiérarchie après des tentatives répétées des clients. Les indicateurs de productivité mesurent le temps de chaque appel, le temps que l'opérateur reste avec le système opérant, le temps d'indisponibilité. Toutefois il n'y a pas d'indicateurs prévus pour analyser le temps de résolution des problèmes, la quantité d'appels reçus avant que le problème soit résolu. De plus, le contrôle de la supervision est plutôt centré sur le respect des procédures et des actions chiffrables et non pas sur la capacité de proposer des solutions aux clients et celle de proposer des améliorations concernant les procédures, et même les processus. Des solutions ainsi envisagées seraient une rupture paradigmatique une fois que la situation actuelle serait cohérente avec le point de vue fonctionnaliste. Les tâches seraient simples et l'activité serait construite par le simple respect des procédures. Le contrôle du temps et le respect des procédures suffiraient pour garantir le volume et la qualité de service prétendue par l'entreprise.

Une nouvelle cohérence serait envisagée par ce changement paradigmatique qui aboutirait à une transformation profonde du travail. Accompagner et résoudre les problèmes des clients seraient une solution pour réduire la fragmentation des processus et proposer des tâches conçues autrement. De nouvelles manières d'évaluer le travail et de nouveaux indicateurs de production seraient néces- 
saires. La composition d'équipes qui seraient responsables d'une partie plus large du processus de production serait envisagée pour accompagner les clients, leurs questions et leurs problèmes. Cette transformation serait accompagnée de nouvelles propositions en ce qui concerne les postes de travail, les systèmes informatisés, l'organisation des espaces de travail. Sans l'adoption des nouveaux paradigmes, les transformations possibles sont étroites et, à terme conduisent à des impasses.

\section{RÉFÉRENCEMENT}

Sznelwar, L.I., Mascia, F.L., \& Bouyer, G. (2006). L'empêchement au travail : une source majeure de TMS ? @ ctivités, 3 (2), 27-44, http://www.activites.org/v3n2/activites-v3n2.pdf

\section{BIBLIOGRAPHIE}

Arbix, G., \& Sznelwar L.I. (2002). Trabalho, controle e impedimento no setor de serviços. In F.J. da Silva, M.S. dos Santos, \& J.I. Rodrigues (Eds.), Crítica Contemporânea (pp. 221-241). São Paulo: Anablume.

Béguin, P. (2005). Concevoir pour les genèses professionnelles. In P. Rabardel, P., \& P. Pastré (Eds.), Modèles du sujet pour la conception : Dialectiques activités développement (pp. 11-29). Toulouse: Octarès Éditions.

Bouisset, S. (1992). Postures et mouvements. In J. Scherrer (Ed.), Précis de physiologie du travail - Notions d'ergonomie (pp. 29-106). Paris: Masson.

Bohle, F., \& Milkau, B. (1991). Vom Handrad zum Bildschirm. München. Apud.: Dejours, C., 1997, O Fator Humano. Rio de Janeiro: Fundação Getúlio Vargas.

Clot, Y. (1995). Le Travail Sans L'homme? Pour une Psychologie des Milieux de Travail et de Vie. Paris: Édition La Découverte.

Clot, Y. (1999). La fonction psychologique du travail. Paris: PUF.

Clot, Y., \& Fernandez, G. (2005). Analyse psychologique du mouvement : apport à la compréhension des TMS. @Activités, 2 (2), 68-78, http://www.activites.org/v2n2/fernandez.pdf

Dejours, C. (1997). O Fator Humano. Rio de Janeiro: Fundação Getúlio Vargas.

Dejours, C. (2000). Nouvelles formes d'organisation du travail et lésions par efforts répétitifs (LER) : Approche de la psychodynamique du travail. In L.I. Sznelwar, \& L.N. Zidan (Eds.), O trabalho humano com sistemas informatizados no setor de serviços (pp. 407-420). São Paulo: Plêiade.

Dejours, C. (2001). Le corps, d'abord. Paris: Payot.

Dreyfus, H., \& Rabinow, P. (1983). Michel Foucault: Beyond Structuralism and Hermeneutics. Chicago: The University of Chicago.

Guérin, F., Laville, A., Daniellou, F., Duraffourg, J., \& Kerguelen, A. (2001). Compreender o trabalho para transformá-lo: A prática da ergonomia. São Paulo: Blucher. (trad. Comprendre le travail pour le transformer - La pratique de l'ergonomie, 1997, LYON:ANACT).

Guyton, A. C., \& Hall, J.E. (1997). Micção, diuréticos e doenças renais. In Tratado de fisiologia médica (pp. 371-386). Rio de Janeiro: Guanabara-Koogan.

Haudricourt, A. G. (1987). La Technologie, Science Humaine. Paris: Maison des Sciences de l'Homme. Apud: Dejours, C., 1997, O Fator Humano. Rio de Janeiro: Fundação Getúlio Vargas.

Jayet, C. (1999). L'homme au travail : entre désir et souffrance. Réflexions d'un médecin du travail. In I. Brunstein (Ed.), L'homme à l'échine pliée (pp. 131-144). Paris: Desclée de Brouwer.

Maggi, B. (2003). De l'agir organisationnel : Un point de vue sur le travail, le bien-être, l'apprentissage. Toulouse: Octarès Éditons. 
Mauss, M. (1934). Les Techniques du Corps. In Sociologie et anthropologie. Paris: PUF. Apud Dejours, C., 1997, O Fator Humano. Rio de Janeiro: Fundação Getúlio Vargas.

Morehouse, L.E. (1958). Human factors philosophy. Human Factors, 1 (1).

Morin, E. (1998). Ciência com consciência. 2 ed. Rio de Janeiro: Bertrand Brasil.

Perilleux, T. (1999). Se donner sans compter. Performance et dépression au travail. In I. Brunstein (Ed.), L’homme à l'échine pliée (pp. 145-160). Paris: Desclée de Brouwer.

Pezé, M.G. (2000). Forclusion du féminin dans l'organisation du travail : un harcèlement de genre. In P. Molinier, \& M.G. Pezé (Eds), Variations sur le corps, Paris: L’Harmattan, Cahiers du Genre, nº 29, pp. $37-52$.

Rabardel, P. (2005) Instrument subjectif et développement du pouvoir d'agir. In P. Rabardel, \& P. Pastré (Eds.), Modèles du sujet pour la conception : Dialectiques activités développement (pp.11-29). Toulouse: Octarès Éditions.

Sznelwar, L. I., \& Massetti. M. (2002). Atteinte corporelle et/ou souffrance psychique, Une étude clinique à partir du vécu des travailleurs souffrant des TMS. Travailler, $\mathrm{n}^{\circ} 8,154-177$.

Zarifian, P. (1999). Objectif compétence. Paris: Éditions Liaisons.

\begin{abstract}
RÉSUMÉ
La discussion sur la genèse des troubles musculo-squelettiques est, en partie, basée sur la question du mouvement, de la vitesse, de la force et de la répétitivité. A partir de différentes études portant sur les TMS concernant des situations de travail dans le tertiaire, nous proposons une discussion basée sur l'idée de l'empêchement. L'analyse est centrée sur l'effort pour ne pas bouger, l'effort pour ne pas penser, l'effort pour ne pas sentir, compte tenu des aspects de l'organisation du travail et du contenu de la tâche qui sont de véritables empêchements pour le sujet.
\end{abstract}

\title{
Mots-CLEFS
}

Empêchement, troubles musculo-squelettiques, analyse ergonomique du travail, centre d'appels.

\section{RESUMEN}

La discusión en torno a la génesis de los trastornos músculo-esqueléticos (TME) se encuentra, en parte, centrada en la cuestión del movimiento, la velocidad, la fuerza y la repetitividad. A partir de diferentes estudios sobre los TME llevados a cabo en el sector terciario, proponemos una discusión basada en la idea de impedimento. Teniendo en cuenta los aspectos de la organización del trabajo y del contenido de las tareas, verdaderos impedimentos para el sujeto, el análisis está centrado en el esfuerzo para no moverse, para no pensar, para no sentir.

Palabras Clave

impedimento, trastornos músculo-esqueléticos, análisis ergonómico del trabajo, centro de llamadas.

Article reçu le 10 Décembre 2004, accepté le 12 Juin 2006 